Ann. Génét. Sél. anim., 1974, 6 (3), 297-303.

\title{
UNE NOUVELLE TRANSLOCATION ROBERTSONIENNE CHEZ LES BOVINS
}

\author{
R. DARRÉ, Hélène-Marie BERLAND et G. QUÉINNEC \\ Chaire de Zootechnie et Économie rurale, \\ École nationale vétérinaire de Toulouse, \\ 31076 Toulouse \\ RÉSUMÉ
}

Une nouvelle translocation robertsonienne notée $F$, différente de celles déjà mentionnées dans l'espèce bovine, a été mise en évidence sur des métis Blond d'Aquitaine $\times$ Limousin. Elle pourrait impliquer un chromosome d'une des paires 7 à II et un chromosome d'une des paires 20 à 25 .

Un même géniteur a été trouvé porteur à la fois de la translocation I-29 et de cette nouvelle translocation.

\section{INTRODUCTION}

Le développement des examens cytogénétiques permet d'envisager progressivement l'existence d'un polymorphisme chromosomique au sein de chaque espèce. Tout se passe comme si la variation génétique représentée essentiellement par le polymorphisme des gènes, sources de l'information, incluait aussi une variation moins fine, appréciable microscopiquement, celle des chromosomes, supports de cette même information.

Certes, l'existence d'une variation du caryotype, dans une même espèce, au sein d'une population d'individus jugés normaux, ne contredit nullement la notion de caryotype normal, ou caryotype de référence, rassemblant les particularités chromosomiques essentielles de 1'espèce considérée. Par ailleurs, les remaniements chromosomiques (inversions, translocations, élongations hétérochromatiques), observées occasionnellement, chez des sujets normaux en apparence doivent être assimilées en première analyse à un réarrangement de la disposition spatiale des loci, sans que soient modifiés nécessairement leur nature et leur dosage. 
L'incidence de tels remaniements sur la nature de l'information génétique est encore à préciser et, de ce point de vue, l'existence d'un effet de position reste une possibilité théorique. Plus évident semble être, dans un certain nombre de cas, un trouble de la reproduction lié à la production de gamètes déséquilibrés, la particularité chromosomique pouvant constituer un obstacle à une réduction méiotique normale.

I,es translocations de type robertsonien occupent une place de choix dans l'étude de ce polymorphisme chromosomique. Ces translocations totales, encore appelées fusions centriques, sont généralement considérées comme des témoins intéressants du processus de spéciation, voire peut-être comme un de ses éléments moteurs dans la mesure où elles ont pu, à l'instar d'autres mutations, participer à l'isolement sexuel, préalable à l'apparition d'une nouvelle espèce.

Par ailleurs, la multiplicité des observations réalisées au sein de diverses espèces, rend compte de la généralité d'un polymorphisme chromosomique intraspécifique fondé en particulier sur le mécanisme robertsonien. L'espèce bovine occupe en 1'occurence une place particulière tant en raison du développement des examens cytogénétiques réalisés dans divers pays, dans le cadre des opérations de sélection et d'échange de géniteurs, que du fait de la fréquence parfois élevée de particularités chromosomiques décelées grâce à un contrôle de plus en plus systématique.

De ce point de vue, la translocation I-29, ainsi dénommée par Gustavsson (I966), est apparemment 1'une des plus répandues dans l'espèce bovine. Ce nouveau chromosome, né d'une fusion centrique entre le plus grand et le plus petit autosome (paires I et 29), a d'abord été décrit dans le bétail Pie-rouge suédois (Gustavsson, r969), puis mentionné à nouveau dans diverses races en de nombreux pays, en France, en particulier (POPESCU, I97I ; DARRÉ et al., I972 ; FroGET et al., I972). A la suite de ces observations, deux nouvelles translocations robertsoniennes ont été identifiées, l'une par Pol,Lock en 1972 sur des bovins Frisons, entre 2 autosomes non homologues provenant des 6 premières paires (2 et 4 selon l'auteur), l'autre par BRUÈRE et CHAPMAN en I973, sur un taureau Simmental néo-zélandais, entre autosomes des paires I I ou I2 d'une part, et I5 ou I6 d'autre part.

Prolongeant cette série d'observations, le cas que nous rapportons ici est celui d'une nouvelle translocation robertsonienne entre chromosomes différents de ceux mentionnés dans les cas déjà décrits.

\section{MATÉRIEL ETT MÉTHODES}

L'étude résulte d'une observation particulière réalisée dans le cadre des examens caryotypiques de taureaux et taurillons d'un Centre d'Insémination Artificielle.

Cinq sujets ont été retenus : le taureau $\mathrm{F}$, sujet principal, son père $\mathrm{V}$, de race Blonde d'Aquitaine, et trois de ses produits. La mère présumée de $\mathrm{F}$, de race Limousine, a dû être écartée après contrôle des groupes sanguins effectué par le Laboratoire Central des Groupes Sanguins du $C . N$. R. Z., son caryotype était normal (60, $\mathrm{XX})$.

Les examens cytogénétiques pratiqués sont fondés sur l'observation de métaphases obtenues à partir de cultures à court terme ( 72 heures) de lymphocytes provenant d'un ensemencement de sang total (méthode de de Grouchy, 1964).

Un idiogramme permettant de classer les chromosomes en fonction de leur longueur relative a été effectué sur 2 sujets, à partir de 5 cellules pour chacun d'eux. Cette longueur relative $l_{r}$ est calculée après projection de photographies de métaphases sur un écran, en faisant le rapport de 
la longueur A de chaque chromosome sur la longueur totale D de la garniture haploïde calculée pour la mitose étudiée.

$$
\mathrm{E}=\frac{\text { somme des longueurs des autosomes }}{2}+\text { longueur du chromosome } \mathrm{X}
$$

Les résultats du contrôle de la descendance et les relevés mensuels des récoltes hebdomadaires de semence du taureau $F$, nous ont été obligeamment fournis par la Coopérative d'Élevage intéressée.

\section{RÉSULTATS}

\section{I. - Mise en évidence des translocations 1-29 et $F$}

Toutes les métaphases provenant du taureau F., ont montré l'existence de 2 chromosomes nouveaux submétacentriques issus de translocations robertsoniennes (fig. I) : l'une identifiée comme étant la $\operatorname{Tr}$ I-29, l'autre notée provisoirement $F$, par allusion à l'animal porteur.
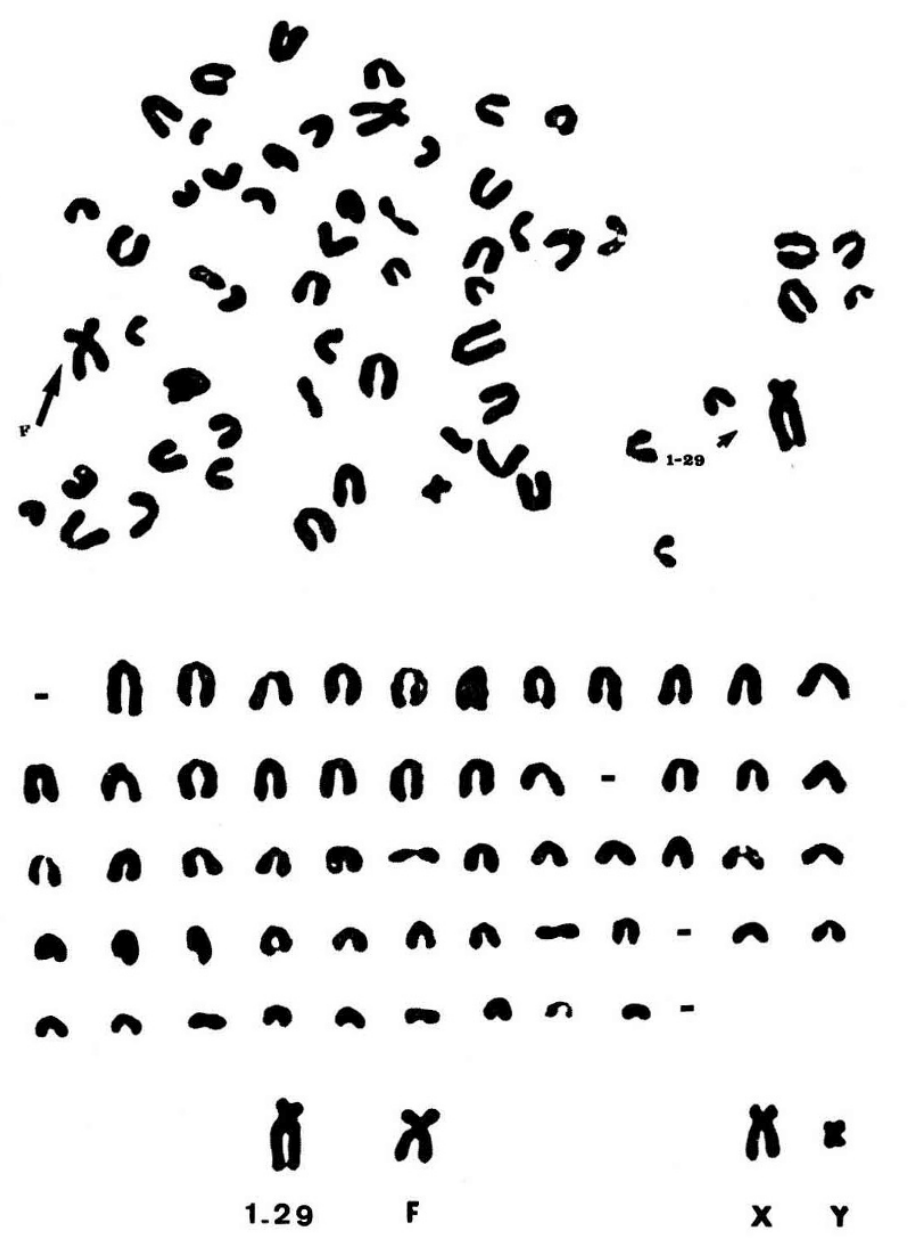

FIG. I. - Caryotype du taureau porteur des translocations $F$ et 1-29 
La translocation I-29 avait déjà été observée sur chacun des 8 autres produits du taureau $\mathrm{V}$ soumis à un examen cytogénétique. Aucun de ces 8 animaux n'était porteur de la translocation $F$. On peut donc, a priori, admettre que le taureau $\mathrm{V}$ avait probablement pour formule caryotypique $58, \mathrm{XY}, 2(\mathrm{I}-29)$.

La translocation $F$ a été retrouvée sur l'une des deux femelles examinées, filles du taureau $\mathrm{F}$, ce qui confirme le caractère constitutionnel et transmissible de la particularité (fig. 2).

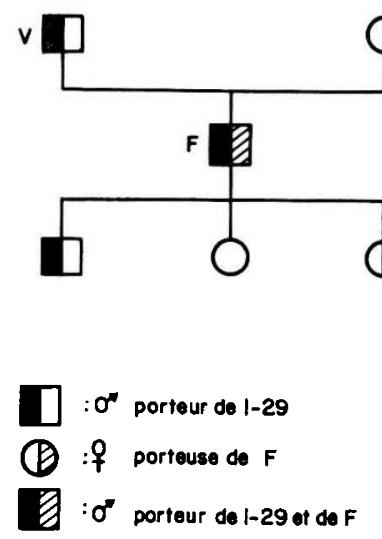

FIG. 2. - Relations de parenté entre animaux porteurs des translocations $F$ et 1-29

\section{2. - Essai d'identification des chromosomes impliqués dans la translocation $F$}

Cinq métaphases provenant du taureau $\mathrm{F}$ et cinq autres provenant de la femelle porteuse ont servi à l'établissement de 2 idiogrammes figurés sur le tableau I. L'erreur standard de la longueur relative n'a été mentionné que pour les chromosomes identifiés avec certitude d'une mitose à l'autre (gonosomes et translocations).

La recherche d'une correspondance entre les bras du chromosome $F$ et un autosome homologue aboutit aux constatations suivantes :

Dans le cas du mâle, les bras longs correspondent à un chromosome de la ro ${ }^{\mathbf{e}}$ paire, et les bras courts à un chromosome de la $2 \mathrm{I}^{\mathrm{e}}$ paire, compte tenu que l'animal est déjà porteur d'une translocation I-29.

Dans le cas de la femelle, les bras longs correspondent à un chromosome de la $9^{\mathrm{e}}$ paire, et les bras courts à un chromosome de la $24^{\mathrm{e}}$ paire.

La méthode ne permet donc qu'une localisation approximative des 2 chromosomes impliqués. Le plus grand proviendrait de l'une des paires numérotées de 7 à II, et l'autre d'une de celles notées de 20 à 25 .

Si l'on considère que les valeurs moyennes, on retiendra qu'il peut s'agir des paires 9 et 22 .

\section{3. - Résultats du contrôle de la descendance}

et appréciation de la fonction sexuelle du taureau $F$

Le taureau $\mathrm{F}$ a été classé $\mathrm{I}^{\mathrm{er}}$, très bon pour la croissance moyenne quotidienne et le poids à âge type de 75 jours dans sa série de testage. Il a été classé $2^{\mathrm{e}}$ bon pour le pointage de la conformation. 
TABLEAU I

Longueurs relatives des chromosomes mesurés chez le taureau et la vache porteurs de la translocation étudiée

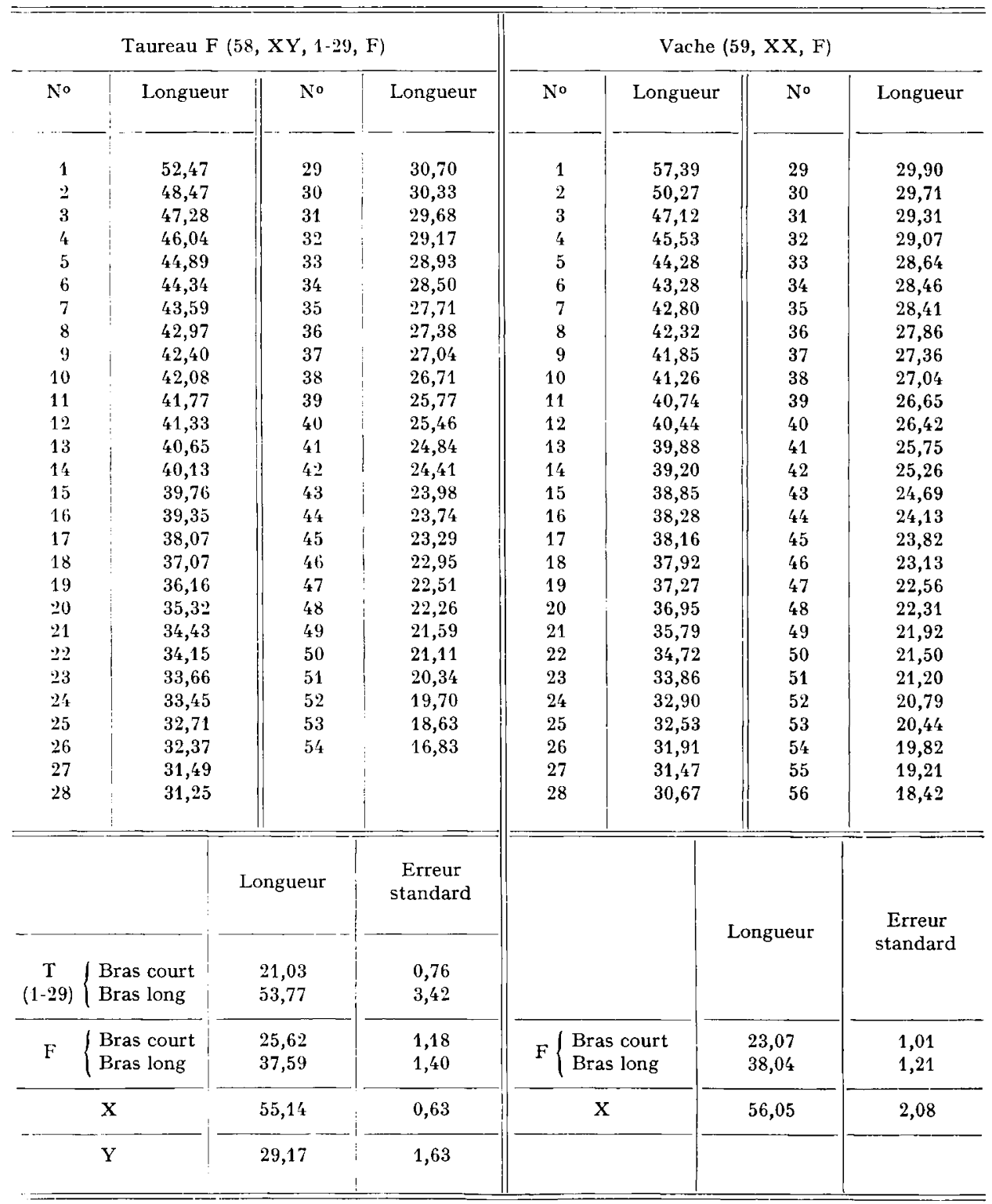


La fonction sexuelle appréciée à partir des 62 récoltes hebdomadaires effectuées de janvier I973 à mars I974 a été jugée moyenne à médiocre pour chacun des caractères habituellement retenus par les centres d'insémination.

\section{DISCUSSION}

La mise en évidence d'une nouvelle translocation robertsonienne chez les bovins pose à nouveau le problème de l'origine et des conséquences éventuelles de ce type de mutation.

La réalisation d'une quelconque translocation robertsonienne transmissible est souvent considérée comme un événement exceptionnel, voire unique (TAYLOR et coll. 1969). En conséquence, la mise en évidence répétée d'une telle mutation résulterait beaucoup plus d'une diffusion à partir d'une origine unique que de mutations récurrentes. Gustavsson (I969) et PoPESCU (I97I), entre autres, trouvent plus plausible l'hypothèse d'une origine commune de la translocation I-29 pour expliquer sa diffusion parmi diverses races bovines européennes à partir d'un ancêtre commun.

Néanmoins, la relative diversité des populations bovines dans lesquelles nous l'avons observée (Blonde d'Aquitaine, Limousine, Charolaise Gasconne, Kouri du Tchad) nous a déjà fait envisager la possibilité d'une mutation récurrente. Sur un plan plus général, BRUÈré (I973) s'appuie sur la constatation, tant chez l'homme que chez les animaux, d'un mosaïcisme associant des cellules normales et des cellules porteuses d'une translocation robertsonienne, pour en déduire que la formation de novo de telles translocations, possible au cours de l'embryogénèse, l'est aussi durant la gamétogénèse. Une éventuelle sélection prézygotique favorable aux gamètes porteurs de l'une de ces translocations a même été invoquée par HAMERTon (I969), dans le cas de l'espèce humaine, ce qui reviendrait à fixer, au moins momentanément, de telles mutations, et à les rendre ainsi repérables. Le phénomène semble enfin avoir une très grande généralité si 1'on considère l'évolution caryotypique de certaines lignées cellulaires marquée par l'apparition progressive de divers types de fusions centriques et la réduction concomitante du nombre des autosomes (NEL,SON REEs et al., I964) (Lithner et PoNTÉn, I966).

Quel que soit le mode d'apparition retenu, la diffusion de ces translocations dans une population naturelle, s'explique assez bien par les mécanismes généraux d'évolution génétique faisant appel, entre autres, à une dérive au sein d'un effectif initialement limité. Dans l'espèce bovine en particulier, l'utilisation prépondérante de certains reproducteurs a pu favoriser la fixation et la diffusion d'une telle mutation apparue fortuitement chez un de ces géniteurs.

Rę̧u pour publication en septembre $19 \%$ t.

\section{SUMMARY}

A NEW ROBERTSONIAN TRANSLOCATION IN CATTLE

A new robertsonian translocation was found in a cytogenetic study of Blonde d'Aquitaine and Limousine cross cattle. 
This new translocation differed from any previously reported in cattle and has been temporarily named $\mathrm{F}$. The two involved chromosomes could belong to 7-II and 20-25 pairs.

One bull was found to be a carrier at the same time of the new F and of I-29 translocations.

\section{RÉFÉRENCES BIBL,IOGRAPHIQUES}

Bruère A. N., Chapman H. M., r973. Autosomal translocations in two exotic breeds of cattle in New Zealand. The Veterinary Record., 92, 6r5-6r8.

1)arré R., Quérnnec G., Berland H. M., I972. La translocation I-29 des bovins. Étude générale et importance du phénomène dans le Sud-Ouest. Rev. Méd. Vet., 123, 477-494.

Frojet J., Coulon J., Nain M. C., Dalbiez J. M., r972. Anomalie chromosomique de type fusion centrique chez un veau Charolais. Bull. Soc. Sci. Vét. Méd. comp. (Lyon), 74, r3r-r35.

Grouchy J. (de), Roubin M., Passage E., I964. Microtechnique pour l'étude des chromosomes humains à partir d'une culture de leucocytes sanguins. Ann. Génét., 7, 45.

Gustavsson I., 1966. Chromosome abnormality in cattle. Nature., 211, 865-866.

Gustavsson I., 1969. Cytogenetics distribution and phenotypic effects of a translocation in Swedish cattle. Hereditas., 63, 68-169.

Lithner F., PontÉn J., I966. Bovine fibroblasts in long-term tissue culture: Chromosome studie. Int. $J$. Cancer., 1, 579-588.

Nelsson-Rees W. A., Kniazeff A. J., Darby N. B., r964. Preservation of bulk chromatin with decrease in number of chromosomes in celles of an established bovine kidney line. J. Nat. Cancer Inst., 33, 347-36r.

Nelsson-Rees W. A., Kniazeff A. J., Darby N. B., I967. Debut and accumulation of centric fusion products : an index to age of certain cell lines. Cytogenetics, 6, 436-450.

Niebuhr E., 1972. Dicentric and Monocentric Robertsonien Translocation in Man. Humangenetik., 16, 2 I7-226.

Pollock D., 1972. A chromosome abnormality in Friesian cattle in Great Britain. Vet. Rec., 91, 309-310.

Popescu C. P., I97r. Deux cas nouveaux de fusion centrique chez les bovins. Ann. Génét. Sél. Anim., 3, 52 I -526.

Taylor K. M., Hungerford D. A., Snider R. L., ig69. Artiodactyl Mammals : their chromosome cytology in relation to patterns of evolution. In K. BENIRSCHKE. Comparative Mammalian Cytogenetics. An International Conference at Darmouth medical Scholl, Hannover New Hampshire, July 29 August 2 I969. Springer, Berlin. 